\title{
INFLUÊNCIA DE DIFERENTES NÍVEIS DE ACETILAÇÃO NAS PROPRIEDADES FíSICO-MECÂNICAS DE AGLOMERADOS E PAINÉIS MADEIRA-CIMENTO
}

Palavras chave: Acetilação

Aglomerados

Painéis madeira-cimento

Histórico:

Recebido 14/02/2013

Aceito 16/12/2013

Keywords: Acetylation

Particleboards

Wood-cement composites

Correspondência: vinnie.castro@gmail.com

DOI:
RESUMO: O uso de partículas de madeira, quimicamente modificadas na produção de painéis de madeira reconstituída, pode, ao mesmo tempo, afetar positivamente a estabilidade dimensional dos painéis, como afetar negativamente a ligação interna. Objetivou-se, neste trabalho, encontrar o ponto ótimo de acetilação para painéis aglomerados e madeira-cimento. Foram utilizadas partículas industriais de Pinus taeda acetiladas a 8,15 e $20 \%$ na produção de aglomerados com resina uréia-formaldeído e corpos de prova de madeira-cimento pelo processo de vibrocompactação. Foram avaliadas as propriedades de absorção de água, inchamento de espessura e ligação interna dos painéis aglomerados de acordo com as normas europeias EN 317 e EN 319. Painéis aglomerados produzidos com partículas acetiladas acima de $15 \%$ apresentaram menores valores de absorção de água e inchamento de espessura. Contudo, o uso da madeira quimicamente modificada afetou negativamente a ligação interna dos painéis. Esse fenômeno pode ser explicado pelo comportamento semelhante da resina com a água, assim, a acetilação em níveis altos inviabiliza a ligação entre adesivo e aderente. Para os corpos de prova de madeira-cimento, a ligação interna melhorou com o aumento dos níveis de acetilação. Porém a inibição à cura do cimento aumentou em partículas com graus de acetilação superior a $8 \%$.

\section{INFLUENCE OF DIFFERENT DEGREES OF ACETYLATION IN THE PHYSICAL AND MECHANICAL PROPERTIES OF PARTICLEBOARDS AND WOOD- CEMENT COMPOSITES}

ABSTRACT: Chemical modified wood particles used to particleboards manufacture may, at the same time, improve the dimensional stability and damage the internal bond. The aim of this research was find the optimal point of acetylation for particleboards. Pinus taeda particles with different degrees of acetylation, 8, 15 and $20 \%$ of weight percentage gain (WGP), were used in the production of particleboards with urea-formaldehyde resin and wood-cement composites produced by mechanical and vibratory compaction. It was evaluated the water absorption, thickness swelling and internal bind of the particleboards according to the European standards EN 317 and EN 319. Particleboards produced with 15 WPG showed the lowest water absorption and thickness swelling values. However, the use of chemically modified wood had a negative influence in the internal bind of the boards. This phenomenon can be explain due to the similar behavior between resin and water, that way, the high degree acetylation stops the adhesive and adherent bind. In the case of wood-cement composites, the internal bind improves as the acetylation degrees get higher. Nevertheless the inhibition of acetylated wood particles to the cement hydration got higher when the WPG was higher than $8 \%$.

Tropical Flora Reflorestadora - Garça, Sáo Paulo, Brasil 


\section{INTRODUÇÃO}

Os painéis de madeira podem ser definidos como produtos compostos de elementos de madeira como lâminas, sarrafos, partículas e fibras, obtidos a partir da redução da madeira sólida e reconstituídos por meio de ligação adesiva (IWAKIRI et al., 2005). Dentre os diferentes tipos de painéis, os de madeira aglomerada assumem elevada importância, pelo fato de não serem exigentes quanto à qualidade da madeira, além de apresentarem flexibilidade de aplicação para os mais variados usos. Contudo, a deficiência na estabilidade dimensional está entre as limitações de desempenho desses painéis. Uma possível estratégia no sentido de melhorar essa propriedade é a modificação química da madeira (IMAMURA et al., 1989).

O processo químico mais utilizado é a acetilação. Esse processo se baseia na estratificação das hidroxilas da madeira por reação com anidrido acético, principalmente na hemicelulose e lignina. Nessa reação, o grupo acetil reage com a hidroxila, formando um éster e ácido acético como subproduto. Como resultado, os grupos hidrófilos da madeira passam a ser bloqueados, ou seja, há uma menor afinidade entre a madeira acetilada e água. Uma vez que a madeira tem sua capacidade de absorção de água reduzida, há um aumento na estabilidade dimensional.

Por outro lado, a formação de painéis de madeira acetilada esbarra em um problema de redução de ligação interna (ROWELL, 1996). Há duas possíveis causas que afetam negativamente essa propriedade: danos físicos às partículas, causados durante o processo de acetilação e interação entre o adesivo e a madeira acetilada.

Segundo Rowell et al. (1987), baixos níveis de acetilação em partículas tipo flake de Populus tremuloides, não chegam a apresentar alterações significativas na estrutura física da madeira. Porém com um grau de acetilação superior a 13,6\%, ocorreu inchamento e fragmentação na parede celular. Com a acetilação em 17,6\%, além da fragmentação, pode ser observada uma redução na espessura da parede celular atribuída à erosão química das camadas $\mathrm{S}_{1}$ e $\mathrm{S}_{2}$.

Além disso, a acetilação também afeta a interação entre $\circ$ adesivo e a superfície da madeira. A resposta à modificação química varia para os diferentes tipos de adesivos. Vick e Rowell (1990) comprovaram que a adesão de 18 diferentes tipos de adesivos foi reduzida de acordo com o grau de acetilação. A maioria dos adesivos não apresentou problemas de ligação a um grau de acetilação de $8 \%$, porém suas propriedades foram afetadas a 14 e $20 \%$.
Um outro tipo de produto reconstituído de madeira com uma composição relativamente simples são os painéis madeira-cimento. Porém entre as principais dificuldades que retardam o desenvolvimento dessa indústria é a seletividade das espécies de madeiras utilizadas.

Em razão da madeira ser um material orgânico, ao ser utilizada na forma de partículas como um agregado do cimento e água, as reações químicas ocorridas pela presença de madeira são desfavoráveis à hidratação do cimento (BIBLIS; LO, 1968). Segundo Latorraca et al. (1999), os componentes orgânicos afetam tanto a possível interação entre a madeira e o cimento, quanto as interações internas do próprio cimento, resultando em painéis de qualidade inferior.

Matsushita et al. (2003) estudaram a composição dos extrativos de Zelkova serrata que inibem a hidratação do cimento. A análise da estrutura química do material isolado indica que tanto o $\mathrm{C}$-glicosidio com quatro grupos hidroxila e a cetona $\alpha$-hidroxilada encontradas na região alifática da molécula podem se ligar aos íons inorgânicos e contribuir para a inibição de sua hidratação. Por essa razão, a modificação química da madeira, por meio do bloqueio de suas hidroxilas é uma possibilidade para a produção de painéis madeira-cimento mais resistentes.

Nesse contexto, no trabalho, apresentaram-se dois principais objetivos: determinar o grau ótimo de acetilação de partículas de Pinus taeda para manufatura de painéis aglomerados com adesivo uréia-formaldeído; e avaliar o efeito da acetilação como pré-tratamento para a redução do efeito inibidor de partículas de Pinus taeda na manufatura de painéis madeira-cimento.

\section{MATERIAIS E MÉTODOS}

\section{Acetilação}

Foram utilizadas, nesta pesquisa, madeira de Pinus taeda, coletada na forma de partículas em uma indústria de painéis aglomerados, localizada no município de Araucária (PR). A granulometria de partículas industriais de mesma origem foi descrita por Trianoski (2010). Tais partículas apresentaram, em média, um comprimento de $6,05 \mathrm{~mm}$, largura de $1,36 \mathrm{~mm}$ e espessura de 0,58 $\mathrm{mm}$.

As partículas, com $3 \%$ de umidade, foram submersas em anidrido acético e submetidas a uma estufa aquecida a $120^{\circ} \mathrm{C}$ por 4,7 e 16 horas para a obtenção do grau de acetilação de 8, 15 e 20\% WPG (weigth percentage gain, que indica o quanto de massa o material ganhou após a modificação química), respectivamente. 
A seguir, as partículas foram lavadas em água corrente e secas em estufa aquecida a $80^{\circ} \mathrm{C}$. Esse método foi indicado por Castro et al. (2013), pela sua eficiência e simplicidade para acetilar partículas de madeiras quando a preservação da cor não é exigida em seu uso final.

\section{Manufatura de painéis aglomerados madeira - resina ureia-formaldeído}

Os painéis aglomerados foram produzidos em escala laboratorial no Laboratório de Painéis de Madeira da UFPR. A resina utilizada foi uréia-formaldeído (UF) aplicada em quantidade de $10 \%$ de sólidos em relação à massa seca das partículas. Resina e parafina ( $1 \%$ de sólido) foram aplicadas sobre as partículas em uma encoladeira tipo tambor rotatório por meio de um bico aspersor integrado ao compressor.

O colchão de partículas foi formado em uma caixa vazada com dimensões laterais de $25 \times 25 \mathrm{~cm}$. $\bigcirc$ cálculo da quantidade de material utilizado para cada chapa foi realizado para uma densidade nominal de $0,75 \mathrm{~g} \cdot \mathrm{cm}^{-3}$ e dimensões finais de $25 \times 25 \times \mathrm{I}, 5 \mathrm{~cm}$. Os painéis foram prensados à temperatura de $160^{\circ} \mathrm{C}$, pressão específica de $40 \mathrm{~kg} \cdot \mathrm{cm}^{-3}$ e um tempo de permanência de 8 minutos. Foram produzidos três painéis por tratamento.

Após período de acondicionamento em câmara climática com temperatura de $20^{\circ} \pm 3^{\circ} \mathrm{C}$ e umidade relativa de $65 \pm 5 \%$, foram retirados de cada painel 16 corpos de prova de $5 \times 5 \mathrm{~cm}$. Destes, 10 foram selecionados para a realização do teste de força da tensão perpendicular, denominado ligação interna (LI) (EUROPEAN COMMITTEE FOR STANDARDIZATION - EN, 1993b) e 6 para o teste de absorção (AB) e inchamento de espessura (IE) após 2 e 24 horas (EN, 1993a).

\section{Teste de inibição da hidratação do cimento}

O teste de inibição da hidratação do cimento foi conduzido antes da produção dos corpos de prova. Foi misturada $15 \mathrm{~g}$ de madeira em forma de pó obtido dos finos produzido pelo moinho de martelo na produção das partículas de aglomerado (que passou na peneira de $1 \mathrm{~mm}$ e ficou retida na de $0,65 \mathrm{~mm}$ ) com $200 \mathrm{~g}$ de cimento Portland CP II - Z e 0,25g de água para cada grama de cimento. A mistura foi colocada em um frasco semiadiabático e para a leitura de temperaturas foram utilizados cabos termopares tipo “J”, conectados a um módulo condicionador de sinais analógicos acoplado a uma placa de conversão analógica-digital por 24 horas.
O cálculo do índice de inibição (I) utilizou a fórmula sugerida por Hofstrand et al. (1984), onde t e t' representam o tempo necessário para atingir a temperatura máxima de hidratação do cimento da mistura de cimento, água e madeira e para a mistura de apenas cimento e água ( $h$ ); $\mathrm{T}$ ' e $\mathrm{T}$ representam a temperatura máxima atingida para mistura cimento água e para a mistura de cimento, água e madeira $\left({ }^{\circ} \mathrm{C}\right)$ e $\mathrm{S}$ ' e $S$ - $(\Delta \mathrm{T} / \Delta \mathrm{t})$ representam o máximo incremento no período de I hora para mistura cimento água e para mistura cimento água madeira:

$I=I 00\left[\left(t-t^{\prime} / t^{\prime}\right)\left(T^{\prime}-T / T^{\prime}\right)\left(S^{\prime}-S / S^{\prime}\right)\right]$

Uma vez obtido o gráfico da reação exotérmica do cimento nas primeiras 24 horas, também foi calculado o fator $C_{A}$, sugerido por Hachmi e Moslemi (1989). Esse fator é a razão entre a área do gráfico da reação com mistura de madeira e a área do gráfico da reação do cimento com água, sem adição de madeira, entre 3,5 horas e 24 horas de reação. Essa área representa a quantidade de calor liberado na reação. Quanto maior o valor do fator, menor a inibição apresentada pela madeira.

\section{Manufatura de corpos de prova de madeira- cimento}

Para a produção dos corpos de prova de madeiracimento, foram utilizadas partículas de Pinus taeda e cimento Portland CP II - Z na proporção I:4, baseada no massa seca do material. Foi adicionada à mistura $40 \%$ de água em relação à massa de cimento, como sugerido por Souza (I994 citado por LATORRACA, 2000).

O método de produção de corpos de prova utilizado foi o de adensamento por vibrocompactação sem o uso de pressão sugerido por Parchen (20l2). A mistura de madeira, cimento e água foi depositada em moldes prismáticos de madeira compensada de $5 \times 5 \times$ $2,5 \mathrm{~cm}$, e submetido à mesa vibratória com uma carga de impacto vertical, equivalente a $220 \mathrm{~N}$ à $3.000 \mathrm{RPM}$, por 30 segundos. Após 7 dias, as formas foram desmontadas e os corpos de prova acondicionados em uma câmara climática à temperatura de $20 \pm 3^{\circ} \mathrm{C}$ e umidade relativa de $65 \pm 5 \%$. Testes de ligação interna (EN, 1993b) foram realizados após 28 dias de cura do cimento.

As análises estatística dos resultados foi realizada conforme delineamento inteiramente casualizado, com emprego de análise de variância e teste de Tukey ao nível de probabilidade de $95 \%$ com auxílio do software Statgraphics Centurion, versão I5.2.06. 


\section{RESULTADOS E DISCUSSÃO}

\section{Painéis aglomerados madeira-resina uréia- formaldeído}

Na Tabela I, mostra-se o efeito dos três níveis de acetilação na estabilidade dimensional e ligação interna.

TABELA I Propriedades físico-mecânicas da painéis aglomerados com diferentes graus de acetilação

TABLE I Physical and mechanical properties of particleboard with different acetylation degrees.

\begin{tabular}{|c|c|c|c|c|c|c|c|}
\hline $\begin{array}{l}\begin{array}{l}\text { Trata } \\
\text { nento }\end{array} \\
\text { nat }\end{array}$ & $\begin{array}{l}\text { WPG } \\
\text { (\%) }\end{array}$ & $\begin{array}{l}\text { Dens. } \\
\left(\mathrm{g} \cdot \mathrm{cm}^{-3}\right)\end{array}$ & $\begin{array}{l}\text { IE 2h } \\
\text { (\%) }\end{array}$ & $\begin{array}{l}\text { IE 24h } \\
\text { (\%) }\end{array}$ & $\begin{array}{c}\text { AB 2h } \\
(\%)\end{array}$ & $\begin{array}{c}\text { AB 24 } \\
(\%)\end{array}$ & $\begin{array}{c}\mathrm{LI} \\
\left(\mathrm{kgf} \cdot \mathrm{cm}^{-2}\right)\end{array}$ \\
\hline $\mathrm{TI}$ & 0 & $0,625^{B}$ & $21,37^{\mathrm{B}}$ & $34,27^{B}$ & $80,33^{A}$ & $114,90^{A}$ & $1788^{\prime}$ \\
\hline T2 & 8 & & & & & & \\
\hline T3 & 15 & & & & & & \\
\hline T4 & 20 & & & & & & \\
\hline
\end{tabular}

* WPG é a porcentagem de ganho de peso após acetilação; IE é o inchamento em espessura após 2 e 24 horas; $A B$ é a absorção de água após 2 e 24 horas; LI é a resistência à tração perpendicular - ligação interna. ** médias seguidas pela mesma letra, dentro da mesma coluna, não se diferenciam estatisticamente ao nível de $5 \%$ de probabilidade pelo teste de Tukey. ${ }^{*} * *$ valores dentro dos parênteses referentes ao Coeficiente de Variação (\%)

Pode se observar que aglomerados produzidos com partículas acetiladas a um nível acima de 15\% (T3 e T4) apresentaram resultados melhores, tanto no inchamento de espessura quanto na absorção de água após 2 e 24 horas de imersão, quando comparados com os aglomerados produzidos com partículas sem ou com baixo grau de acetilação ( $T$ I e T2). Esse resultado pode ser atribuído ao processo de acetilação, no qual o anidrido acético reage com os grupos hidrófilos da parede celular da madeira, conforme relatado por Rowell (2006).

Madeira - $\mathrm{OH}+\mathrm{CH}_{3} \mathrm{C}(=\mathrm{O})-\mathrm{O}-\mathrm{C}(=\mathrm{O})-\mathrm{CH}_{3} \rightarrow$ (madeira) (anidrido acético)

Madeira - $\mathrm{O}-\mathrm{C}(=\mathrm{O})-\mathrm{CH}_{3}+\mathrm{CH}_{3} \mathrm{C}(=\mathrm{O})-\mathrm{OH}$ (madeira acetilada) (ácido acético)

O bloqueio dessas hidroxilas impede a ligação entre moléculas de água e as partículas de madeira, refletindo diretamente nas propriedades físicas inchamento de espessura e absorção de água dos painéis de madeira aglomerada com resina UF.
Por outro lado, a adesão entre ureia-formaldeído e a madeira é um fenômeno físico-químico similar à absorção de água (PIZZI, 1994). Da mesma maneira que a acetilação inibe a ligação entre as hidroxilas e moléculas de água, também afeta negativamente a interação entre adesivo e madeira. $O$ efeito prático dessa interação pode ser observado nas médias encontradas para a propriedade de ligação interna. Quanto maior o grau de acetilação das partículas, menor à resistência à tração perpendicular dos painéis.

Entretanto, os valores médios de ligação internas obtidos para todos os tratamentos avaliados atendem ao requisito mínimo da norma europeia para aglomerados que é de $3,57 \mathrm{kgf} \cdot \mathrm{cm}^{-2}$ (EN, 2003). Com relação aos resultados apresentados na literatura, Iwakiri et al. (20/2) e Trianoski et al. (20l3) obtiveram, para painéis de Pinus taeda e UF, valores médios de 8,77 e $7,14 \mathrm{kgf} . \mathrm{cm}^{-2}$, respectivamente. Portanto, os resultados de ligação interna obtidos para painéis de madeira com grau de acetilação abaixo de 15\% ( TI, T2 e T3) estão compatíveis com os obtidos para a madeira estudada.

\section{Corpos de prova de madeira-cimento}

Na Figura I, apresentam-se as curvas de reação exotérmica de cura do cimento misturado somente com água e quando misturados com madeira de Pinus taeda sem acetilação $(0 \%)$ e com ganhos de peso de 8,15 e $20 \%$ após acetilação.

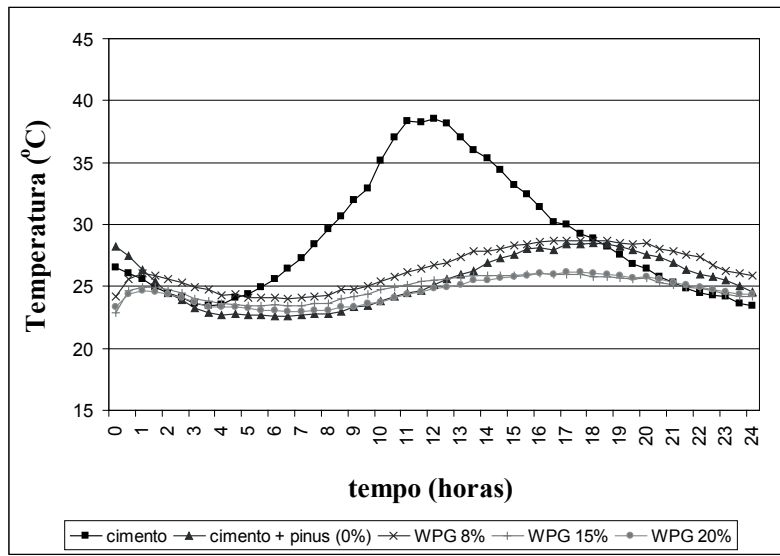

FIGURA I Efeito do grau de acetilação da madeira na curva de hidratação do cimento.

FIGURE I Effect of the acetylation degree of wood on the cement hydration curve.

$\mathrm{Na}$ tabela 2, estão apresentados os valores encontrados nas curvas de hidratação do cimento mostradas na figura $\mathrm{I}$ e os resultados médios de 
densidade dos painéis e ligação interna dos corpos de prova. Nesta tabela, também estão apresentadas as propriedades de densidade e ligação interna dos corpos de prova produzidos com cimento e madeira de Pinus taeda acetilada a 0, 8 , 15 e $20 \%$.

TABELA 2 Fatores de inibição na cura do cimento misturados à madeira acetilada e valores de densidade e ligação interna dos compósitos madeira-cimento.

TABLE 2 Inhibition factors of cement - acetylated wood mixture and values of density and internal bonding of wood-cement composites

\begin{tabular}{cccccc}
\hline $\begin{array}{c}\text { Trata } \\
\text { mento }\end{array}$ & $\begin{array}{c}\text { WPG } \\
(\%)\end{array}$ & $\begin{array}{c}\text { Indice de } \\
\text { Inibição } \\
(\%)\end{array}$ & $\begin{array}{c}\text { Fator } \mathrm{C}_{\mathrm{A}} \\
(\%)\end{array}$ & $\begin{array}{c}\text { Densidade } \\
\left(\mathrm{g} \cdot \mathrm{cm}^{-3}\right)\end{array}$ & $\begin{array}{c}\text { Ligação } \\
\text { Interna } \\
\left(\mathrm{kgf} \cdot \mathrm{cm}^{-2}\right)^{*}\end{array}$ \\
\hline $\mathrm{Cl}$ & 0 & 8,62 & 85,7 & $0,704^{\mathrm{A}}$ & $17,27 \mathrm{I}^{\mathrm{A}}$ \\
$\mathrm{C} 2$ & 8 & 10,29 & 89,4 & $0,780^{\mathrm{AB}}$ & $47,345^{\mathrm{B}}$ \\
$\mathrm{C} 3$ & 15 & 11,09 & 83,7 & $0,810^{\mathrm{AB}}$ & $51,716^{\mathrm{B}}$ \\
$\mathrm{C} 4$ & 20 & 13,89 & 82,9 & $0,892^{\mathrm{B}}$ & $74,354^{* * *}$ \\
\hline
\end{tabular}

* Valores de ligação interna ajustados para uma densidade de $0,772 \mathrm{~g} /$ $\mathrm{cm}^{3}$." Médias seguidas pela mesma letra, dentro da mesma coluna, não se diferenciam estatisticamente ao nível de $5 \%$ de probabilidade pelo teste de Tukey. $*$ *** O rompimento ocorreu na madeira de Pinus taeda em que o corpo de prova estava colado. Esse resultado sugere que $\circ$ valor da ligação interna do tratamento $C 4$ foi superior à $74,354 \mathrm{kgf} / \mathrm{cm}^{2}$.

De acordo com a classificação sugerida por Hofstrand et al. (1984), o Pinus taeda é uma espécie de baixa inibição à hidratação do cimento, ou seja, apresenta um índice inferior à 10\%. O uso da madeira acetilada aumenta a inibição inicial da hidratação do cimento. Contudo a acetilação da madeira tende a aumentar o índice de inibição para valores acima de 10\%. Entretanto, se considerada a classificação sugerida por Hachmi e Moslemi (1989) baseada no fator $\mathrm{C}_{\mathrm{A}}$, $\circ$ aumento na inibição da hidratação do cimento ocorre somente em partículas acetiladas com grau acima de $8 \%$.

Embora os índices que indicam a compatibilidade da madeira com o cimento não sejam alarmantes, ao se produzir compósito de madeiracimento com a madeira sem tratamento prévio, obteve-se um bloco com baixíssima ligação interna. $O$ aumento do nível de acetilação melhorou esse valor, ao ponto de que corpos de prova produzidos com $20 \%$ de madeira acetilada suportarem carga superior ao da madeira sólida de Pinus taeda usada como suporte para a realização do ensaio. Uma possível explicação para esse fenômeno é o de que a baixa absorção das partículas acetiladas permite que $\circ$ cimento tenha maior disponibilidade de água, tornando sua cura mais eficiente com o tempo. O grau ótimo de acetilação das partículas foi o de $8 \%$ (tratamento $\mathrm{C2}$ ), onde foi observado o melhor fator $C_{A}$, ou seja, o menor grau de inibição à hidratação do cimento e ainda uma melhora significativa na ligação interna, quando comparada com compósitos feitos com partículas não-acetiladas.

\section{CONCLUSÕES}

A acetilação de partículas de madeira com anidrido acético é um processo simples, que não necessita aquisição de grandes equipamentos, e pode ser utilizada na fabricação de painéis reconstituídos.

Painéis aglomerados produzidos com uréiaformaldeído e partículas acetiladas com I5\% de ganho de peso apresentam melhores resultados quanto à estabilidade dimensional. Porém, $\circ$ uso de maiores graus de acetilação não é recomendado, em razão da redução acentuada da propriedade de ligação interna das partículas.

Por outro lado, o uso de madeira acetilada na produção de painéis de cimento-madeira é promissor. O uso de partículas acetiladas a $8 \%$ reduziram a inibição quanto à hidratação inicial do cimento e melhoraram, consideravelmente, os resultados de ligação interna. A acetilação de madeira pode ser usada como um prétratamento das partículas antes da produção de painéis madeira-cimento.

\section{REFERÊNCIAS}

BIBLIS, E. J.; LO, C. Effect on the setting of southern pinecement mixtures. Forest Products Journal, Madison, v. 18, n. 8, p. 28-34, Aug. 1968.

CASTRO, V. G.; KLOCK, U.; IWAKIRI, S.; MUNIZ, G. I. B. Avaliação colorimétrica de partículas de Pinus taeda submetidas a diferentes métodos de acetilação. Scientia Forestalis, Piracicaba, v. 4I, n. 98, p. 265-270, jun. 2013.

EUROPEAN COMMITTEE FOR STANDARDIZATION. EN 3 I : particleboards, specifications. Bruxelas, 2003.

EUROPEAN COMMITTEE FOR STANDARDIZATION. EN 3 17: aglomerado de partículas de madeira e aglomerado de fibras de madeira: determinação do inchaço na espessura depois da imersão em água. Bruxelas, 1993a.

EUROPEAN COMMITTEE FOR STANDARDIZATION. EN 319: aglomerado de partículas de madeira e aglomerado de fibras de madeira: determinação da força da tensão perpendicular sobre a superfície plana da placa. Bruxelas, 1993b.

HACHMI, M.; MOSLEMI, A. A. Correlation between woodcement compatibility and wood extractives. Forest Products Journal, Madison, v. 39, n. 6, p. 55-58, June 1989. 
HOFSTRAND, A. D.; MOSLEMI, A. A.; GARCIA, J. F. Curing characteristics of wood particle from nine northern Rocky Mountain species mixed with Portland cement. Forest Products Journal, Madison, v. 34, n. 2, p. 576I, Feb. 1984

IMAMURA, Y.; SUBIYANTO, B.; ROWELL, R. M.; NILSSON, T. Dimensional stability and biological resistance of particleboard from acetylated Albizzia wood particles. Wood Research, Kyoto, n. 76, p. 49-58, 1989.

IWAKIRI, S.; ANDRADE, A. S.; CARDOSO JÚNIOR, A. A.; CHIPANSKI, E. R.; PRATA, J. G.; ADRIAZOLA, M. K. O. Produção de painéis aglomerados de alta densificação com uso de resina melamina-uréia-formaldeído. Cerne, Lavras, v. II, n. 4, p. 323-328, out./dez. 2005.

IWAKIRI, S.; MATOS, J. L. M.; TRIANOSKI, R.; PRATA, J. G. Produção de painéis aglomerados homogêneos e multicamadas de Melea azedarach (Cinamomo) e Pinus taeda com diferentes teores de resina. Cerne, Lavras, v. I8, n. 3, p. 465-470, jul./set. 2012.

LATORRACA, J. V. F. Eucalyptus spp. na produção de painéis de cimento-madeira. 2000. 191 p. Tese (Doutorado em Ciências Florestais) - Universidade Federal do Parará, Curitiba, 2000.

LATORRACA, J. V. F.; IWAKIRI, S.; LELIS, R. C. Efeito inibidor de cinco espécies florestais sobre a cura do compósito cimento-madeira. Floresta e Ambiente, Seropédica, v. 6 , n. I, p. 76-82, jan./dez. 1999.

MATSUSHITA, Y.; MIYAWAKI, S.; YASUDA, S. Manufacture of wood-cement boards VIII: cement-hardening inhibitory compounds of keyaki (Japanese zelkova, Zelkova serrata). Journal of Wood Science, Amsterdam, v. 49, n. 3, p. 28I284, June 2003.
PARCHEN, C. F. A. Compósito madeira cimento de baixa densidade produzido com compactação vibro dinâmica. 2012. I72 p. Tese (Doutorado em Ciências Florestais) - Universidade Federal do Parará, Curitiba, 2012.

PIZZI, A. Urea-formaldehyde adhesives. In: Advanced wood adhesives technology. New York: M. Dekker, 1994. p. 19-66.

ROWELL, R. M. Acetylation of wood, journey from analytical technique to commercial reality. Forest Products Journal, Madison, v. 56, n. 9, p. 4-12, Sept. 2006.

ROWELL, R. M. Physical and mechanical properties of chemically modified wood. In: HON, D. N. S. (Ed.). Chemical modification of lignocellulosic materials. New York: M. Dekker, 1996. p. 295-310.

ROWELL, R. M.; YOUNGQUIST, J. A.; SACHS, I. B. Adhesive bonding of acetylated aspen flakes, part I: surface changes, hydrophobicity, adhesive penetration and strength. International Journal of Adhesion and Adhesives, Guildford, v. 7, n. 4, p. 183-188, Oct. 1987.

TRIANOSKI, R. Avaliação do potencial de espécies florestais alternativas, de rápido crescimento, para produção de painéis de madeira aglomerada. 2010. 262 p. Dissertação (Mestrado em Ciências Florestais) Universidade Federal do Parará, Curitiba, 2010.

TRIANOSKI, R.; IWAKIRI, S.; MATOS, J. L. M.; CHIES, D. Utilização da madeira de Cryptomeria japonica para produção de painéis aglomerados. Scientia Forestalis, Piracicaba, v. 4I, n. 97, p. 57-64, mar. 2013.

VICK, C. B.; ROWELL, R. M. Adhesive bonding of acetylated wood. International Journal of Adhesion and Adhesives, Guildford, v. 10, n. 4, p. 267-272, Oct. 1990. 